\title{
Signaling Networks: Information Flow, Computation, and Decision Making
}

\section{Evren U. Azeloglu and Ravi lyengar}

Department of Pharmacology and Systems Therapeutics and Systems Biology Center New York, Mount Sinai School of Medicine, New York, New York 10029

Correspondence: ravi.iyengar@mssm.edu

\section{SUMMARY}

Signaling pathways come together to form networks that connect receptors to many different cellular machines. Such networks not only receive and transmit signals but also process information. The complexity of these networks requires the use of computational models to understand how information is processed and how input-output relationships are determined. Two major computational approaches used to study signaling networks are graph theory and dynamical modeling. Both approaches are useful; network analysis (application of graph theory) helps us understand how the signaling network is organized and what its information-processing capabilities are, whereas dynamical modeling helps us determine how the system changes in time and space upon receiving stimuli. Computational models have helped us identify a number of emergent properties that signaling networks possess. Such properties include ultrasensitivity, bistability, robustness, and noise-filtering capabilities. These properties endow cell-signaling networks with the ability to ignore small or transient signals and/or amplify signals to drive cellular machines that spawn numerous physiological functions associated with different cell states.

\section{Outline}

1 Introduction

2 Emergent properties of signaling networks

3 Information flow and processing
4 Concluding remarks

References

Editors: Lewis Cantley, Tony Hunter, Richard Sever, and Jeremy Thorner

Additional Perspectives on Signal Transduction available at www.cshperspectives.org

Copyright $\odot 2015$ Cold Spring Harbor Laboratory Press; all rights reserved; doi: 10.1101/cshperspect.a005934 


\section{INTRODUCTION}

Coordinated regulation of cellular processes allows cells to maintain homeostatic balance and make decisions as to whether to divide, differentiate, or die. In each case, the cell responds to chemical, mechanical, or electrical signals, including hormones, neurotransmitters, mechanical stretch and shear, and ion currents. The signaling pathways activate relay information to effectors that alter subcellular processes, but they also process this information. Information processing involves computation in which the network of connected signaling molecules recognizes the amplitude and duration of the incoming signal and produces an output signal of appropriate strength and duration. This depends on the organization (topology) of the signaling network and can change the relationship between inputs and outputs. For example, the cell can limit responses to maintain homeostatic balance or trigger a set of changes that takes it to another state (e.g., differentiation or division). The inherent computational abilities of signaling networks thus provide the cell with decision-making capabilities.

In all signaling pathways, information flows through coupled biochemical reactions and molecular interactions to the cellular machines that control its output: biochemical (e.g., metabolic enzymes), mechanical (e.g., actin cytoskeleton contractility), or electrical (e.g., ion channel activity in excitable cells). Most of these pathways are part of wider networks (Weng et al. 1999). Studying the flow of information (signal) through these networks (see Box 1) is like studying traffic patterns, which depend on the population density of the towns through which the highways pass, the state of local roads, the time of the day, the weather, and other factors. Similarly, to understand the flow of signals that regulate cellular machines, we must know how the pathways involved are connected to form networks, the characteristics of the connections, and how information is processed as it flows through these interconnected pathways. Below, we discuss the organization of these networks and explain how they can be modeled quantitatively.

\subsection{Versatility of Signaling Components Enables Pathways to Form Networks}

Most signaling pathways in mammalian cells interact with one another (Jordan et al. 2000). This so-called "crosstalk" starts at the level of receptors. Growth factor receptors with intrinsic tyrosine kinase activity, for example, interact with multiple effector pathways, including the Ras-ERK-kinase pathway (Morrison 2012), the phosphoinositide 3-kinase
(PI3K) pathway (Hemmings 2012), and the phospholipase-C $\gamma$ pathway (Fig. 1A) (Bootman 2012). Similarly, in the case of receptors coupled to heterotrimeric $\mathrm{G}$ proteins, the ability of both the G $\alpha$ and $G \beta \gamma$ subunits to transmit signals allows the receptors to couple to multiple downstream effectors (Fig. 1B).

Small G proteins, such as Ras, Rho, Rap, and Cdc42, are also major loci of interconnectivity (Bar-Sagi and Hall 2000). Their guanine nucleotide exchange factors (GEFs) and GTPase-activating proteins (GAPs) can be regulated by numerous mechanisms, including binding of ligands such as cAMP, calcium, and diacylglycerol (DAG), or posttranslational modifications such as phosphorylation and acylation. Multiple receptors feed into these GTPase regulators, and the small G proteins themselves can modulate the activity of multiple signaling pathways by targeting several different effectors (Fig. 1C).

Protein kinases and protein phosphatases are additional loci at which multiple signaling pathways interact. Typically, a single protein kinase or phosphatase can be activated by multiple receptors and has multiple substrates. Together, all these components make the signaling pathways within a cell extensively interconnected.

Most mammalian proteins are present as multiple isoforms. These can arise from alternative splicing of a single mRNA or use of alternative initiation codons, or they can be products of different genes altogether. Typically, these isoforms have different characteristics that alter both upstream and downstream interactions (connectivity) and/or their intracellular localization. This further increases connectivity. An early example is adenylyl cyclases (AC1-AC8), which allow signals from multiple types of receptors and ion channels to feed into the cAMP pathway (Pieroni et al. 1993; Sassone-Corsi 2012). These enzymes can be activated or inhibited by signals relayed via different types of receptors (Fig. 2), thus enabling cAMP levels to provide an integrated measure of the information coming into the cell from various sources. cAMP in turn works through multiple effectors (e.g., protein kinase A, the EPAC Rap-GEF, and the cyclic-nucleotide-gated channels) to regulate numerous cellular targets to evoke physiological responses.

Why is one class of signaling component often responsible for regulating many cellular machines? We do not yet know the answer. The cellular machinery probably evolved to produce distinct activity patterns in response to specific signals, whose amplitude, duration, and subcellular location evoke signature responses from only a subset of cellular machines. There is suggestive evidence to support this idea but no definitive proof, and this is an area of ongoing research in systems biology. 


\section{BOX 1. INFORMATION AND SIGNALS IN CELL-SIGNALING PATHWAYS}

In the context of intracellular-signaling pathways and networks, the terms information and signal are used interchangeably. These terms are most often used to distinguish the activity state of a signaling component. Biochemical information, or signal, flows from component A to component B as the activity state of A controls the activity state of component $B$. The activity state of a given molecule is defined as the fraction of active (e.g., $\mathrm{A}^{*}$ or $\mathrm{B}^{*}$ ) to total molecules (e.g., A or B). A typical node $\mathrm{A}$ in this example could be a receptor, such as the adrenergic receptor, that has an interaction (i.e., edge) with a target node $B$, such as the $G$ protein Gs. Communication through this edge results in node $\mathrm{A}$ activating node $\mathrm{B}$ (i.e., $\beta$ adrenergic receptor-activating Gs). It is important to note that information content at any given time depends on both the local concentration of interacting components and the reaction kinetics that govern change in activity state. Green arrows in the figure denote information flow.

\section{Biochemical information flow}
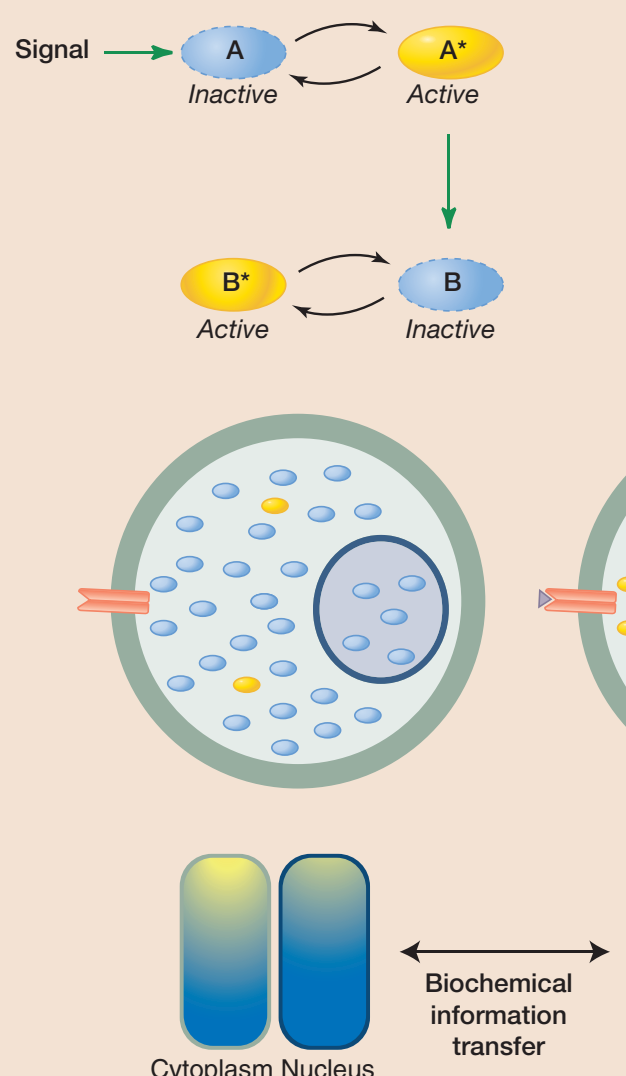

Cytoplasm Nucleus

\section{Spatial information flow}
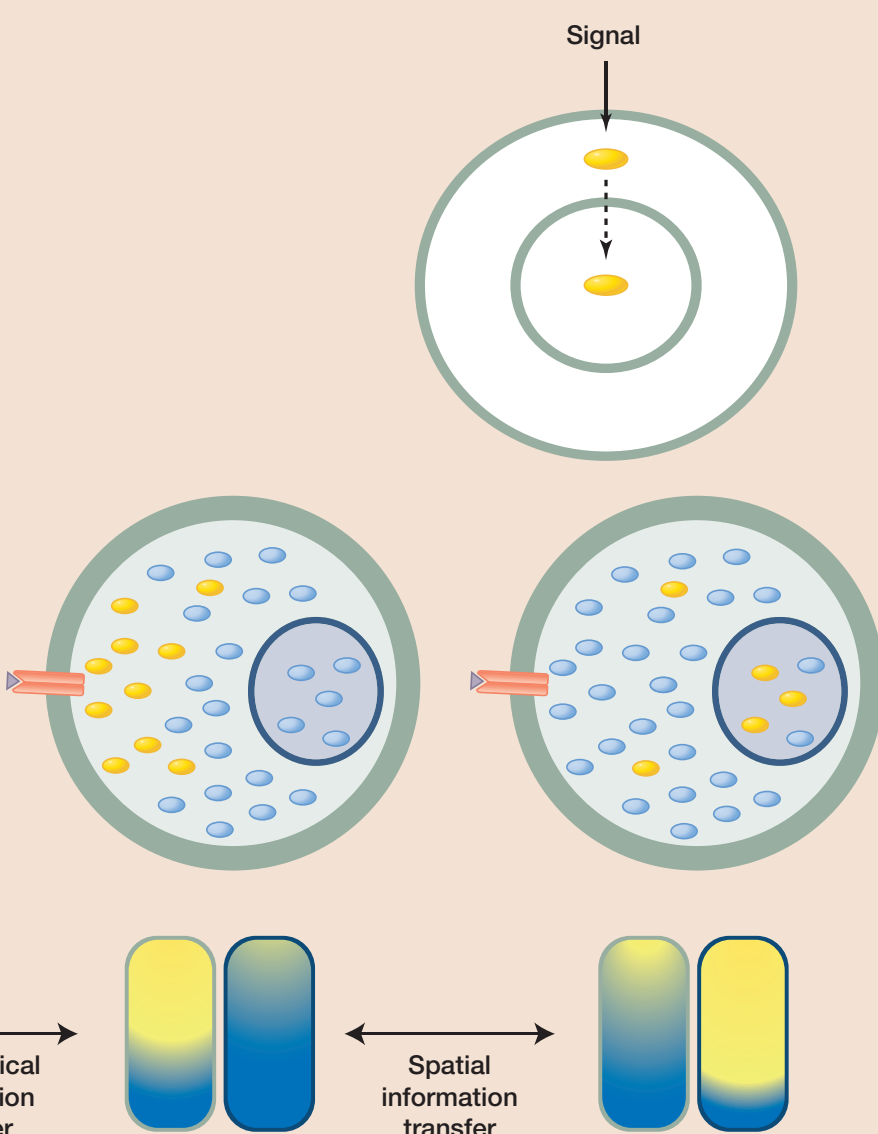

CytoplasmNucleus

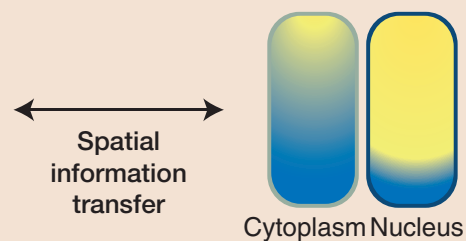

Cytoplasm Nucleus

Biochemical information transfer is established through coupled reactions, which are either noncovalent reversible binding reactions or enzymatic reactions that involve covalent modification. However, such information flow sometimes results in the movement of components from one subcellular compartment to another. The most common examples of such movements are the translocation of protein kinases or transcription factors from the cytoplasm to the nucleus in response to hormone or growth factor signals, where they interact with other proteins or DNA to affect gene expression. This is considered spatial information transfer. 
A

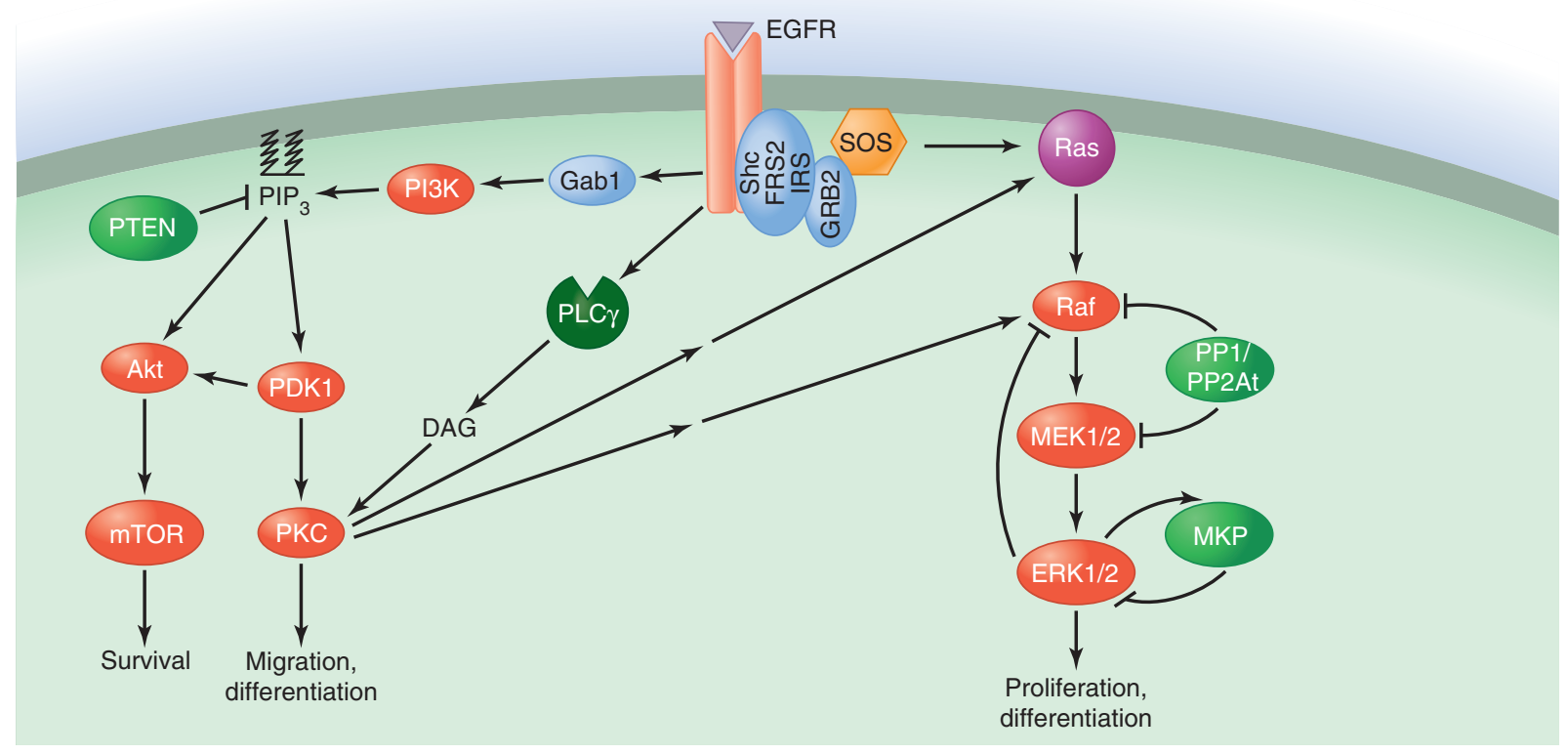

B

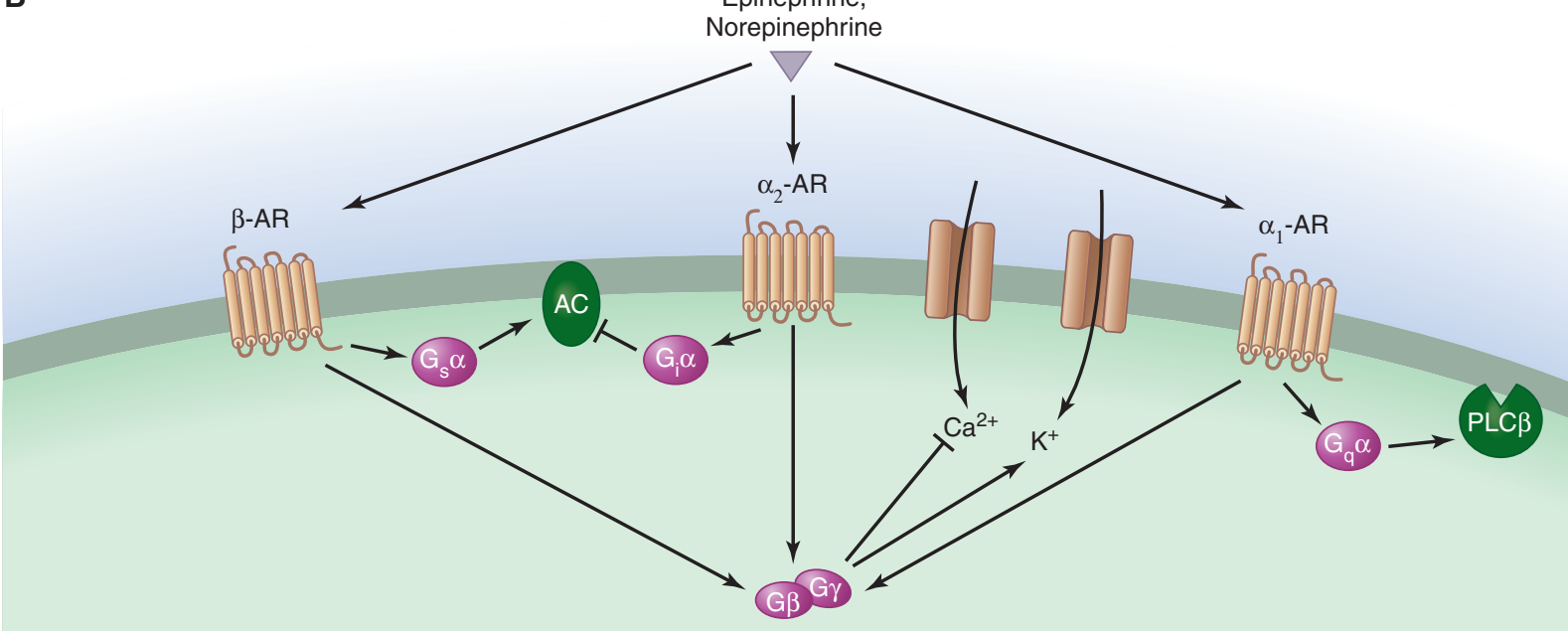

C

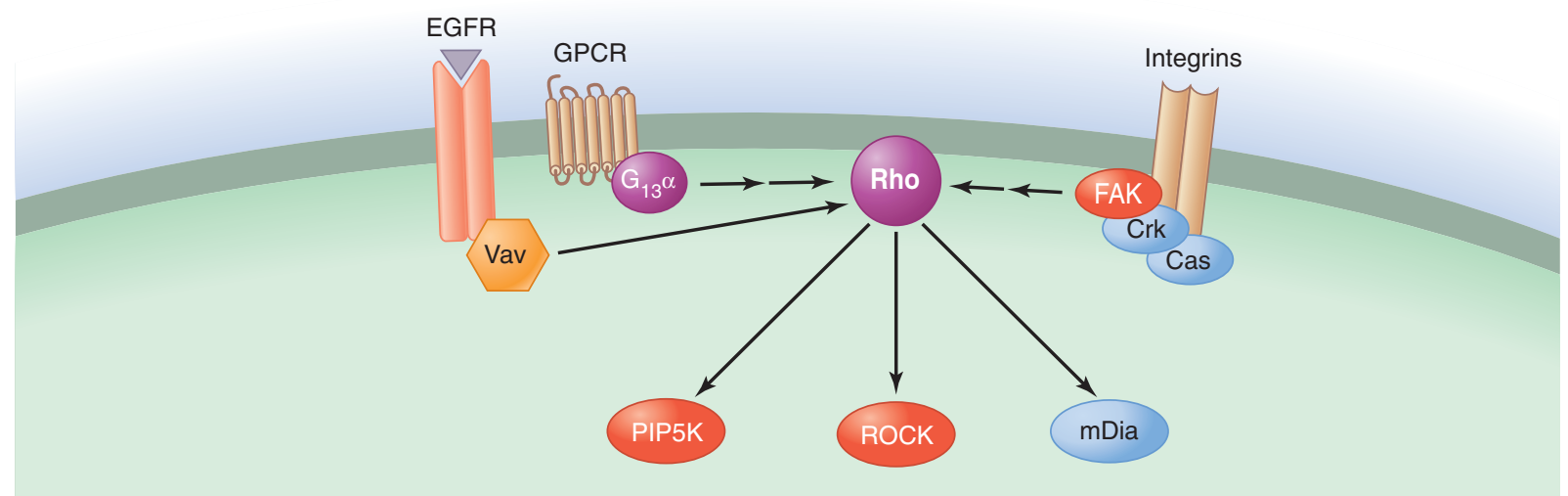

Figure 1. Interaction of multiple components with receptors leads to signal flow within multiple signaling pathways. Good examples are the interplay of PI3K, PLC $\gamma$, and Ras ERK signaling following activation of epidermal growth factor receptor (EGFR) (A), heterotrimeric $\mathrm{G}$ proteins linked to adrenergic receptors (ARs) $(B)$, and the small $\mathrm{G}$ protein Rho $(C)$. 


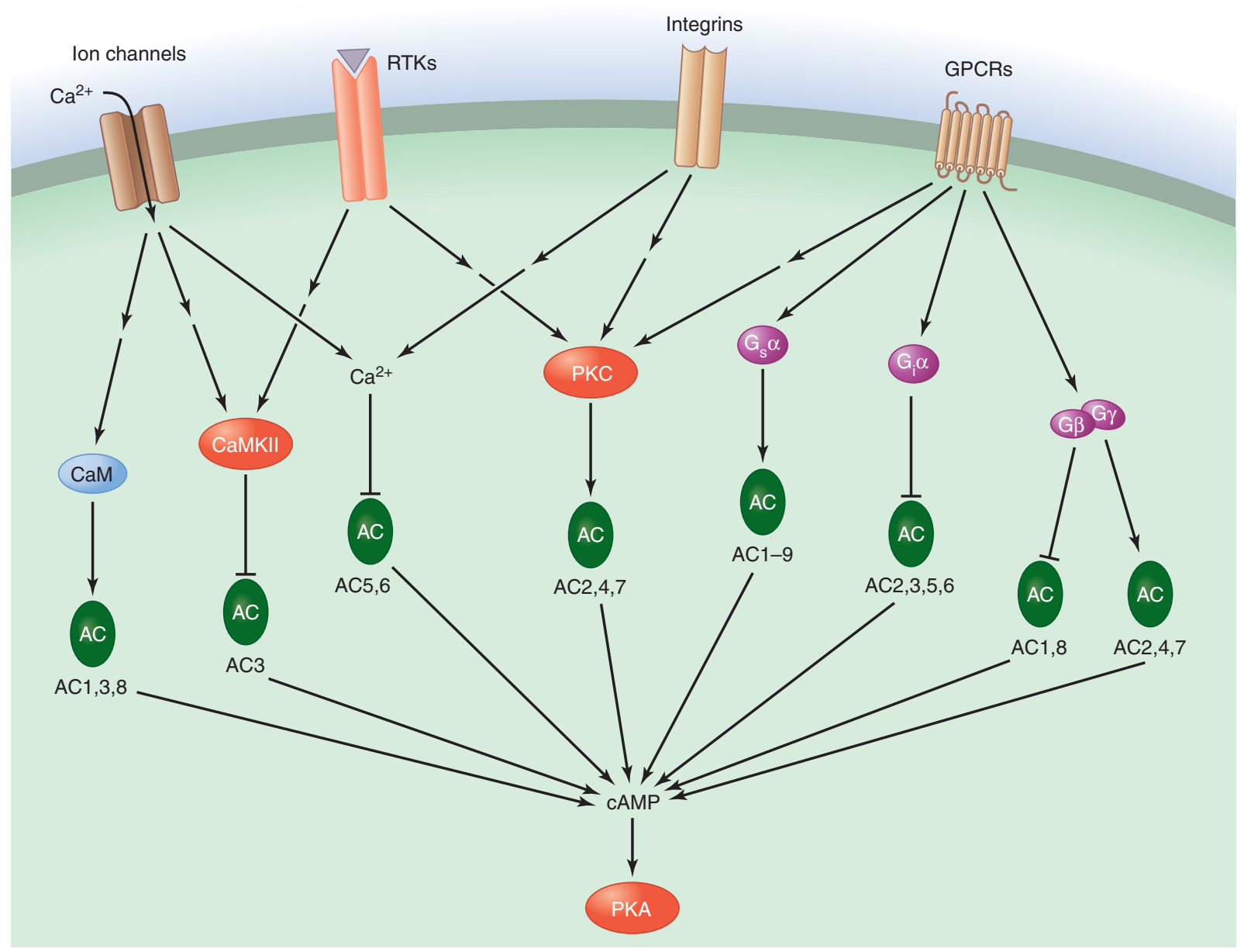

Figure 2. Different adenylyl cyclase (AC) isoforms are activated by multiple different upstream signals. This allows the second messenger cAMP to provide an integrated measure of information from several different sources.

\subsection{Graph-Theory-Based Models Allow Us to Infer the Information-Processing Ability of Signaling Networks}

The complexity of signaling networks requires that we have an orderly approach if we are to understand what these networks do, and how they do it. For this, in addition to experiments, we need a theoretical framework. Networks are often studied using a branch of mathematics called graph theory that analyzes systems composed of objects (called nodes or vertices) and binary relationships (called edges). First developed by Euler in 1736 (Biggs et al. 1986), graph theory focuses on the combinatorial study of multiple interactions between numerous components, and it has been very successfully used in many disciplines, including social sciences. A classic experiment by Stanley Milgram in 1967 using chain letters showed that any two persons in the United States are connected by a median of five interconnected individuals and led to the concept of "six degrees of separation" (Pool et al. 1989). Statistical analyses have since defined metrics that provide estimates of overall connectivity and organization within networks. In general, these focus on computing the relationships between edges and nodes within the network (see Box 2).

Signaling networks, like many other networks, are scale-free. This means the ratio of edges to nodes follows a power law rather than a Gaussian distribution (Barabasi and Albert 1999). In a typical network, there are a small number of nodes with many edges, called hubs. In contrast, most nodes have only few edges. Distribution of highly and sparsely connected nodes is not evenly spaced. Interconnectivity between nodes is also not evenly distributed with the overall networks. A metric called the clustering coefficient can be used to compute the extent of interconnectivity. This is used to identify highly or sparsely connected regions within a network, which allows us to identify clusters or subnetworks within the larger network (see Box 2) 


\section{BOX 2. COMPUTATIONAL MODELS OF SIGNALING NETWORKS}

The complexity that arises from having many isoforms of signaling components, and their differential connectivity, makes it hard to intuitively understand the organization of signaling networks and to make accurate predictions of their outputs a priori. However, computational analysis based on experimental observations could yield accurate predictions while improving our understanding of the signaling landscape. Two types of computational approaches are useful: (1) network models, and (2) dynamical models.

\section{Network Models}

Network analysis, application of a field of mathematics called graph theory, typically involves the use of statistical methods to identify characteristics of a network. So-called network models incorporate an additional level of complexity, the relationship between the different entities. Although statistical and network models share many features in terms of methods used for analysis, the outcomes are different. Statistical models provide knowledge about relationships within a system by analyzing the experimental background for significant correlative changes. Network models provide knowledge about the organization of the system, in addition to identifying the underlying relationships. The term used to describe network organization is topology. The organization of networks can be categorized based on how the components are connected to one another. There are different types of networks. In part B in the figure below, three different networks are shown in decreasing order of clustering coefficients. Small-world networks have densely connected topologies, in which the typical distance between two randomly chosen nodes is proportional to the logarithm of the number of nodes (Barabasi and Albert 1999).

The connectivity between components may be direct (i.e., component A chemically interacts with component B) or indirect (wherein changes in component $\mathrm{A}$ modulate changes in component $\mathrm{C}$ without direct chemical interactions). Direct binary relationships allow tracking of pathways from receptors to effectors, and prediction of distal relationships. The use of models of network topology to predict the information-processing capability of the system relies on identification of small organizing units, called network motifs, such as feedback and feedforward loops. The smallest fundamental motifs have two to four interacting components; some of the motifs that are prominently featured in biological networks are outlined in part B in the figure below.

A

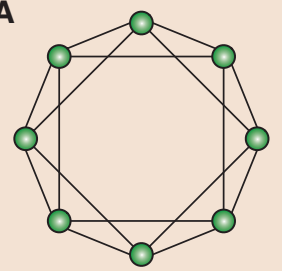

Regular

B

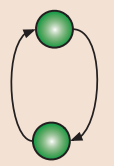

Two-node positivefeedback loop

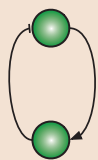

Two-node negativefeedback loop

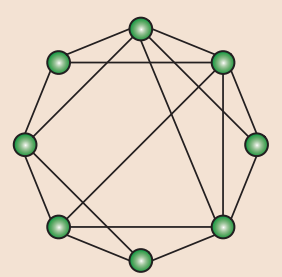

Small-world

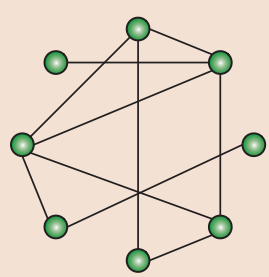

Random
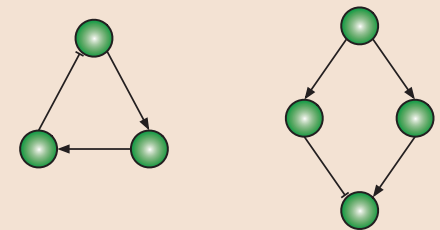

Two-node doublenegative-feedback loop

Three-node negativefeedback loop

Four-node incoherent feedforward motif

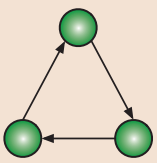

Three-node positivefeedback loop 
A limitation of graph-theory-based analysis is that it provides a static view of the system. It tells us how the system is organized, but does not tell us how such organization enables the system to change with respect to time on receiving a signal. In signaling networks, every connection between components in a signaling reaction represents one or more chemical reactions, whose dynamics are regulated by both the concentration of the reactants and the rates of the forward and reverse reactions. Accordingly, connectivity between components does not automatically imply that signals are propagated through them. To understand how spatiotemporal reaction dynamics affect signal propagation, we need a second type of analysis called dynamical modeling.

\section{Dynamical Models}

Dynamical models describe how a system changes with respect to time, based on reaction rates between components. They can be divided into two classes: (1) deterministic models, in which the time-dependent change in the state of the system can be predicted from the initial concentrations of the reactants and the reaction rates; and (2) stochastic models, in which the trajectory of the system is not fully predictable but depends on the probability of a set of reactions occurring. Typically, stochastic models involve systems in which the concentrations of reactants or the volumes occupied are very small, which decrease or increase the certainty of interactions, respectively.

Deterministic models usually use ordinary-differential-equation (ODE)-based representations of biochemical or biophysical interactions. ODE systems are based on the assumption that system dynamics depend only on time. These offer a detailed and quantitative picture of interactions. Using the known parameters of reaction stoichiometry, a system of ODEs can be formulated to represent the flow of information within a signaling network. These equations can then be simultaneously solved as a function of time to reveal the

Ordinary differential equations (ODEs)

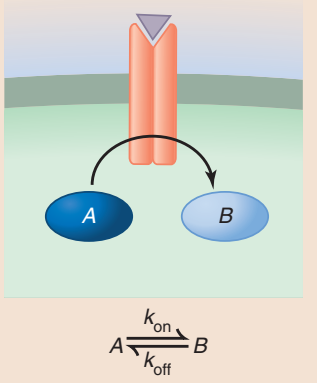

Partial differential equations (PDEs)

$$
\begin{aligned}
& \frac{d A}{d t}=k_{\text {off }}[B]-k_{\text {on }}[A] \\
& \frac{d B}{d t}=k_{\text {on }}[A]-k_{\text {off }}[B]
\end{aligned}
$$

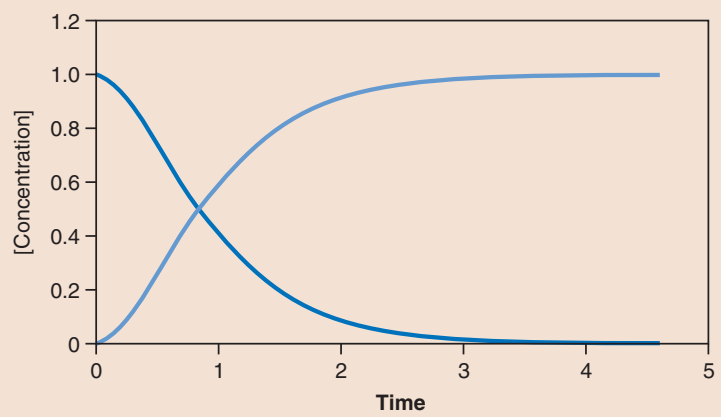

$\frac{\partial A}{\partial t}=D_{A}\left(\frac{\partial^{2} A}{\partial x^{2}}+\frac{\partial^{2} A}{\partial y^{2}}\right)-k_{\text {on }}[A]+k_{\text {off }}[B]$

$\frac{\partial B}{\partial t}=D_{B}\left(\frac{\partial^{2} B}{\partial x^{2}}+\frac{\partial^{2} B}{\partial y^{2}}\right)-k_{\text {off }}[B]+k_{\text {on }}[A]$
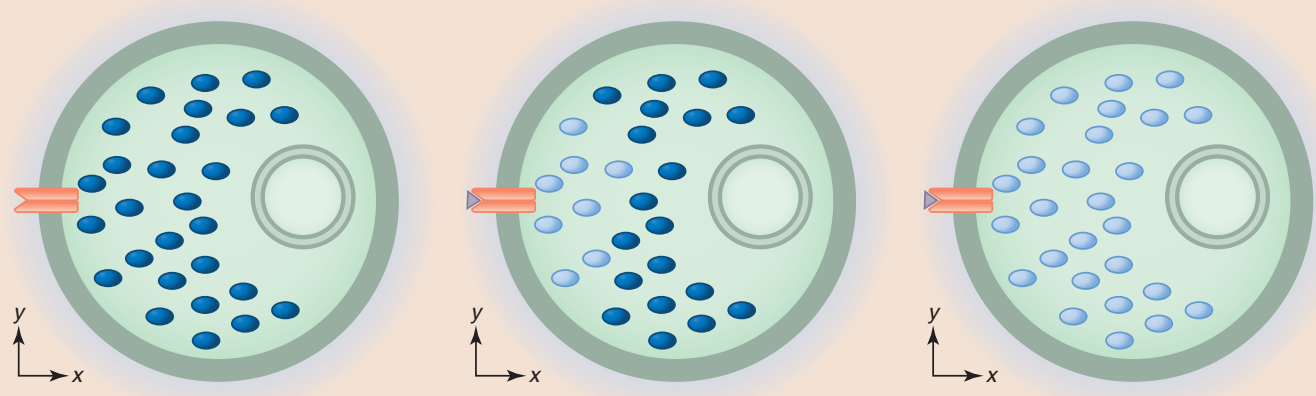

Time, $t=0$

Time, $t=1$

Time, $t=2$ 
behavior of individual components as the reactions proceed (see figure above). A typical ODE model can be used to describe signal flow upon receptor ligand activation.

Although ODE-based models are common and very valuable as a first approximation, they suffer from an inherent simplifying assumption that all components are evenly distributed within the reaction space (i.e., time is the only independent variable). This is usually not true within cells. Simple ODE models can be improved by introducing spatial information transfer via multiple subcellular compartments such that the rates at which components move between compartments are specified. Typically, for a signal flowing from the cell surface to the nucleus, the cell can be thought of as a three-compartment system comprising the plasma membrane, cytoplasm, and the nucleus.

A more sophisticated, and complete, way of improving deterministic models is to use partial differential equations, in which each component has two associated attributes: specific reaction rates $(k)$ and a diffusion coefficient $(D)$, which define system behavior as a function of time $(t)$ and space $(x, y)$, respectively (see figure above). For example, a model of signal flow from receptor tyrosine kinases to extracellular-regulated kinase (ERK) to transcription factors involves information flow from the cell surface membrane through the cytoplasm to the nucleus, and it is best described by a partial-differential-equation (PDE)-based model. Although such models provide a more realistic picture of the cell, they have some drawbacks. For example, diffusion coefficients for most cellular components have not been measured and they need to be approximated from molecular weights. In addition, numerical simulations of PDE-based models remain computationally expensive; so such models are not widely used. Nevertheless, to fully understand how signaling reactions occur in localized regions within the cell (especially for cells with nonstandard geometries) one must use PDE-based models

Sometimes reactions in cells involve components that are present in very small numbers or do not react in an easily predictable manner. The most common example is the binding of a transcription factor to the promoter of a gene. To model such an event accurately, we need to consider the probability of the interaction occurring as a function of time, volume, and number of molecules. Stochastic models incorporate these parameters, and use Gillespie's algorithm as the standard approach for computations (Gillespie 2007). These models combine individual probabilities of interactions at the molecular level under given circumstances (e.g., probability of binding of a transcription factor to a promoter given its concentration and volume constraints). In theory, they can provide highly accurate representations of biochemical events; however, because of the need for a large number of operations per unit time, they tend to be computationally expensive even with small spatiotemporal constraints. In addition, because of their random nature, in most cases, they require Monte Carlo simulations that use random sampling to determine how the system changes with respect to time or space. Such models are also often difficult to develop owing to a paucity of experimental data. Hence, stochastic models are not as commonly used as deterministic models to describe large systems but could become more popular in the future as more information becomes available.

The local topology within a signaling network can have numerous effects on its input-output relationships. Signals may be amplified, dampened, prolonged or shortened; such behavior is controlled by ubiquitously repeating patterns of connectivity called regulatory motifs. Several classes of regulatory motifs are observed in biological networks (Alon 2007). These include feedforward and feedback loops and bi-fan motifs (see Box 2).

The feedforward motif has two arms from a starting node to a target node. A good example is the signal from the MAP kinase ERK to the transcription factor Fos (Fig. $3 \mathrm{~A}$ ). ERK stimulates transcription of the Fos gene and consequently production of Fos protein, which it can also phosphorylate. Because the phosphorylated form of Fos is protected from degradation, the feedforward loop prolongs the signal from the ERK pathway. Extending signal output is one feature of a feedforward motif. Another is redundancy, which can make a system robust against perturbations (see below). Feedforward loops in which all the relationships are of the same sign (e.g., all stimulatory) are called coherent feedforward loops, and they can be used for additive computation within the signaling network. When two arms of the starting node have opposing effects, the motif is called an incoherent feedforward loop. Incoherent feedforward loops can induce accelerated transient output following a step input (Mangan and Alon 2003), which could be useful for rapid cellular response.

Feedback loops are common regulatory features of biochemical pathways and networks. They can be both positive and negative. In negative-feedback loops, a downstream component inhibits an upstream stimulator. This type of regulation is very common in metabolic pathways, where a downstream metabolite will allosterically inhibit an upstream enzyme to reduce flux. It is also found in signaling. Phosphorylation and deactivation of the $\beta$-adrenergic receptor by PKA to limit the duration of responses to the hormone epinephrine is a classic example (Fig. 3B). Positive-feedback loops are also found in signaling pathways. 
A

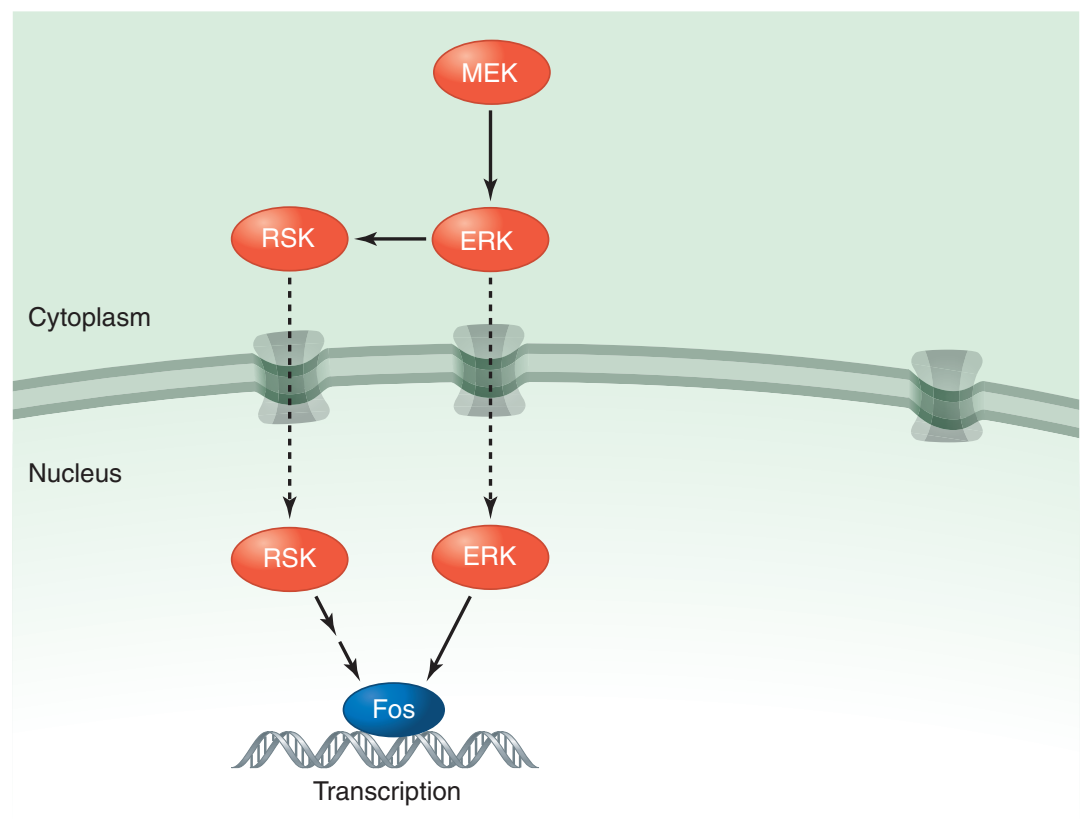

C

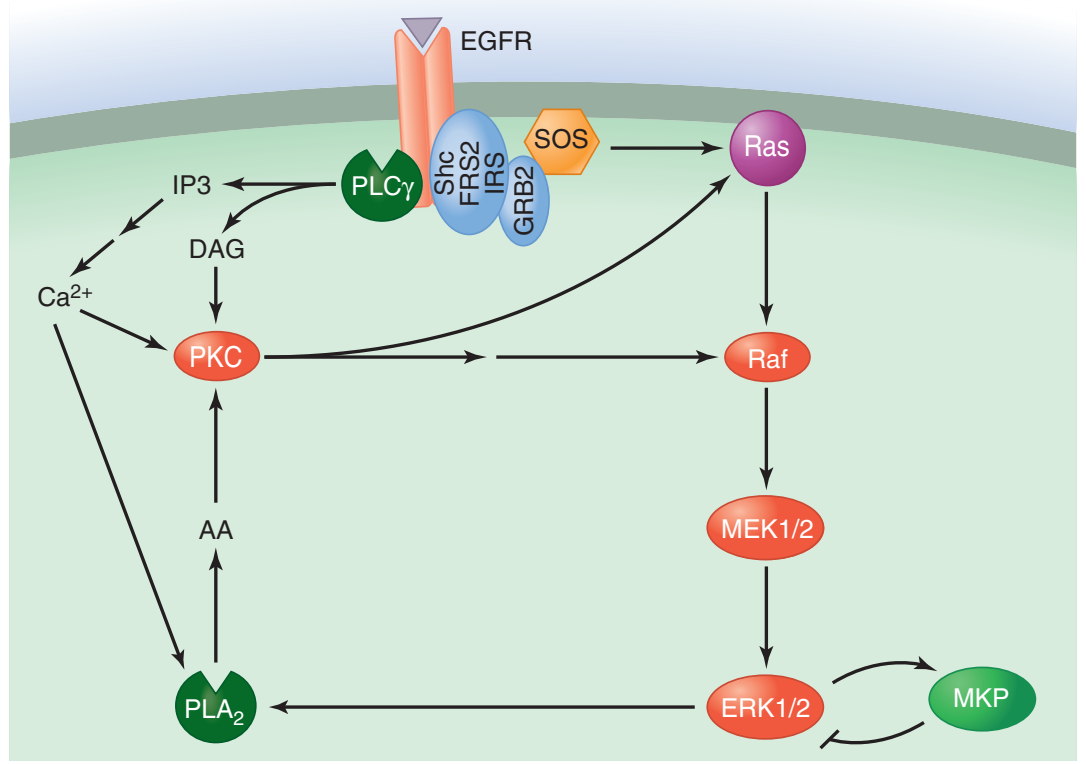

B

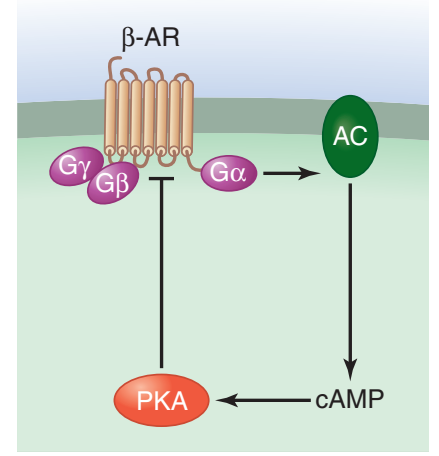

D

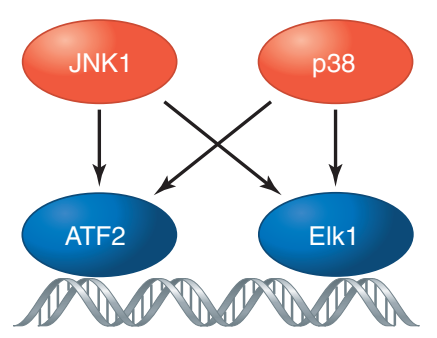

Figure 3. Regulatory motifs in cell signaling networks. (A) A representative coherent feedforward motif depicting signal flow from ERK to the transcription factor Fos, where both transcriptional activation and posttranslational stabilization lead to prolonged signal duration. (B) Negative feedback through $\beta$-adrenergic receptor $(\beta$-AR)-activated PKA limits the duration of response. $(C)$ Positive-feedback loops within ERK-PLA2-PKC pathways can lead to bistability. $(D)$ Kinases JNK and p38 phosphorylate and activate both of the transcription factors ATF2 and Elk1, forming a bi-fan motif that provides tight regulation, including temporal control and coincidence detection.

An example is the interaction between ERK and phospholipase A2 (PLA2), which results in the activation of PLA2 (probably via activation of MNK1/2, which phosphorylates PLA2 at S727), leading to the increased production of arachidonic acid. Diacylglycerol and arachidonic acid to- gether can activate protein kinase C (PKC). This positivefeedback loop results in prolonged activation of PKC as well as ERK (Fig. 3C). Such positive-feedback loops can function as switches to move the signaling network from an inactive to persistently active state. 
Bi-fan motifs form another class of abundant motifs found in signaling networks, especially at the interface between protein kinases and transcription factors. Most protein kinases phosphorylate multiple transcription factors, and many transcription factors are phosphorylated by multiple protein kinases. The JNK and p38 MAP kinases and the transcription factors ATF2 and Elk1 are shown in Figure 3D. This crisscrossing allows a cell to integrate multiple signals to give a cohesive pattern of gene expression. In the case of Elk1 and ATF2, for example, the motif enables upstream kinases to effectively synchronize the activity levels of the transcription factors.

\section{EMERGENT PROPERTIES OF SIGNALING NETWORKS}

The organization of a signaling pathway and its regulatory motifs can endow a signaling network with emergent properties that the individual components by themselves do not possess. These are difficult to predict from examination of any one of the components.

\subsection{Ultrasensitivity}

Sometimes a stimulus, such as receptor activation, can produce a switchlike response in a downstream signaling component. In such a system, at a certain point called the threshold, a small change in the ligand/receptor can cause a large change in the activity of a downstream effector. Such responses are called ultrasensitive. This was first observed with coupled enzymes switching between activity states (Goldbeter and Koshland 1981). Subsequently, similar ultrasensitivity has been observed in control of the cell cycle by the ERK pathway, where small changes in stimulus cause large changes in ERK activity (Ferrell and Machleder 1998). Ultrasensitivity can be produced by several mechanisms such as cooperativity, multistep regulation as seen in the ERK pathway, and by changing the levels of activators to inhibitors of a signaling component such as a GTPase (Lipshtat et al. 2010).

\subsection{Bistability}

The ability of positive-feedback loops like the one involving PKC, ERK, and PLA2 (Fig. 3C) to function as switches is an emergent property called bistability. ODE-based models (see Box 2) of the ERK-PLA2-PKC pathway (Bhalla and Iyengar 1999) allow one to plot the activity of ERK as a function of protein kinase $\mathrm{C}$ activity and vice versa (Fig. 4). The two curves intersect three times. In this system, if the initial stimulus is above the threshold level (the middle intersection point) needed to increase activity of either protein kinase $\mathrm{C}$ or ERK, the system will move from

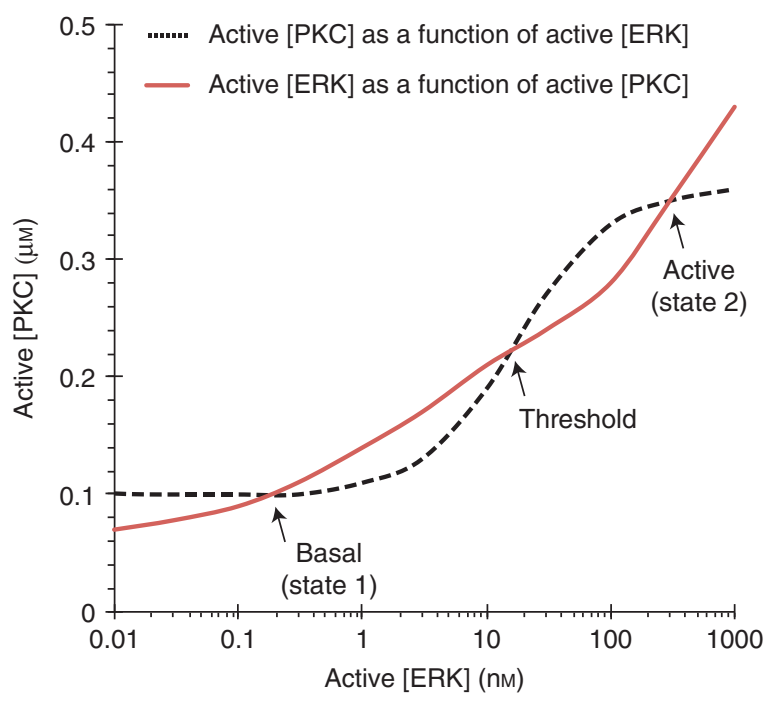

Figure 4. Activity states of components in a positive-feedback loop can be plotted as functions of each other to study the characteristics of a bistable system that switches from an inactive basal state (state 1) to an active state (state 2 ) once a threshold is reached. For example, when the concentration of active MAPK or PKC is above the threshold levels upon growth factor receptor activation, the system moves to state 2 . The plot is obtained by computational modeling of the positive-feedback loop in Figure 3C, and recording the steady-L-state levels of active PKC for a given fixed concentration of active MAPK (black dashed line) upon stimulation, and vice versa (red solid line).

the lower intersection point (basal state) to the upper intersection point (active state) for a prolonged period. The upper and lower intersection points represent the two stable states. The middle intersection point represents the level above which the initial stimulus engages the feedback loop and moves the system to the activated equilibrium state. Otherwise, when the stimulus is withdrawn the system will revert to its basal equilibrium state. The computational modeling analysis shows that the connectivity alone is insufficient for the switching behavior. The concentrations of the signaling components and the reaction rates are critical in ensuring that a feedback loop with this topology functions as a bistable switch.

Bistability is involved in triggering numerous biological processes. The positive ERK-PLA2-PKC-feedback loop described above functions as a switch in cerebellar neurons, where it converts brief electrical signals into long-lasting changes in cellular responsiveness called long-term depression (Tanaka and Augustine 2008). In another example, during cell replication, a positive-feedback loop confers bistability on cyclin B expression, enabling the cell to enter mitosis (Sha et al. 2003). Similarly, programmed cell death is controlled by two cooperative positive-feedback loops, which confer bistability on mitochondrial permeability initiated by caspase-3 (Bagci et al. 2006; Green and Llambi 2014). 


\subsection{Redundancy and Robustness}

Because most signaling pathways are critical for survival, unsurprisingly, cells often use multiple pathways to connect a single input with an output. For example, activated epidermal growth factor receptors (EGFRs) simultaneously activate PKC (via tyrosine phosphorylation of PLC $\gamma$ and generation of diacylglycerol [Newton 2014]) and the small G protein Ras (via formation of Grb2-SOS adapter-GEF complexes), both of which feed into the ERK pathway (Morrison 2012). As shown in Figure 1A, this redundancy provides a safety net for activation of key processes that are necessary for cell survival or homeostasis. It further allows signaling networks to withstand perturbations that can alter input-output relationships, and it can ensure that cells respond only to stimuli of appropriate duration and magnitude, increasing robustness.

Network topology plays an important role in the robustness of signaling systems. Coherent feedforward motifs (see Box 2), for example, can be used in transcriptional regulatory networks as "persistence monitors" that respond to only sustained stimuli (Mangan and Alon 2003). Biochemical properties of signaling components such as a high catalytic rate or the ability to be altered by posttranslational modification are also major contributors to robustness of signaling networks.

\subsection{Oscillatory Behavior}

Oscillatory phenomena occur in cellular events from cell division to circadian rhythms. They generally involve negative-feedback loops; coupled positive- and negative-feedback loops can also lead to sustained oscillations (Tyson and Novak 2010). The cell cycle, for example, requires the cyclic expression and degradation of cyclins (Xiong 2013). Control of the cell cycle in Xenopus oocytes was one of the first oscillatory biological systems to be modeled with numerical simulations (Novak and Tyson 1993). Two feedback loops, one on top of the other, operate in the Xenopus oocyte cell cycle. In this case, a negative-feedback loop is necessary and sufficient for the oscillations of the driver, cyclin-CDK complex, but a positive-feedback loop dampens and synchronizes the cycles and is necessary for systems-level physiological operations (Pomerening et al. 2005).

\section{INFORMATION FLOW AND PROCESSING}

\subsection{Information in Signaling Network} Is Contextual

Although most cells share a multitude of common signaling components, identical signals can lead to diverse biological responses from different cell types. For instance, a pathway could have different outputs in two different cell types because they have different numbers or types of receptors.

A good example is a small peptide hormone known by two names: vasopressin (VP) and antidiuretic hormone $(\mathrm{ADH})$. The two names arise because the hormone has very different physiological effects in two different cell types. In smooth muscle cells, it binds to V1 receptors that couple through the $\mathrm{G}$ protein $\mathrm{Gq}$ and calcium to regulate contractility, leading to contraction of blood vessels. In kidney cells, the same hormone binds to V2 receptors that couple through Gs and cAMP to regulate water uptake, leading to an antidiuretic effect. Thus, information in the hormone molecule is very differently interpreted by the two different cell types in different tissues in the body.

Such contextual dependence of information can occur within the cell as well. For example, in neurons, cAMP can bind to and activate PKA, leading to effects on metabolic enzymes, gene expression, and channel activity. cAMP can also directly bind to and gate a class of cation channels called HCNs. Because the affinity of cAMP for the HCN channels is substantially less than its affinity for PKA and because these channels are largely present in the distal dendrites, the information in cAMP can be selectively transmitted to metabolic enzymes and gene regulation without substantially affecting the electrical behavior of the neurons. In effect, cAMP at low concentrations represents information for PKA but no information for HCN channels, because the lower affinity precludes the channels from binding cAMP. In considering information flow within the signaling network, we therefore always need to know the characteristics of both the transmitter of information and the receiver.

\subsection{Information Transmitted Can Be Proportional to the Stimulus, Processed, or Dissipated}

Often the subcellular localization of the components of a signaling network and their relative affinities ensure that information flows proportionally (e.g., linearly) from receptor to effector. Typically, when the strength of the extracellular signal is such that only a small proportion of receptors are activated, the resultant downstream responses are proportional to the level of receptor activation. In some cases, however, network topology, spatial localization of the signaling components, and biochemical characteristics of individual components can lead to information processing. Enzymatic reactions, for example, often amplify signals, depending on the catalytic rate of the enzyme and its ability to activate multiple targets (Fig. 1B). Conversely, signal flow that involves adaptor proteins or other binding reactions such as scaffold proteins is less likely to amplify 
information because these interactions are stoichiometric. Adaptors and scaffolds play a critical role in signal processing, however, adding spatial constraints to information flow and defining bidirectional specificity. Network topology such as positive-feedback loops and coherent feedforward loops can increase the amplitude as well as the duration of output. Amplification by any of these mechanisms can increase sensitivity of the response or reduce responses to small or transient stimuli.

For certain signaling pathways that are ultrasensitive, dissipation of biological information is just as important as amplification. Prolonged signaling may lead to cell death or disease states. Drug-induced calcium excitotoxicity, which kills neurons, and mutated signaling proteins that lead to neoplasia, provide good examples of the deleterious effects of aberrant signals. Hence, signal dissipation is critical for proper functioning of signaling pathways. A range of negative regulators act on receptors, GTPases, effectors, and protein kinase signals to control and dissipate them before they can reach downstream effectors and produce physiological responses. Receptors are negatively regulated by protein kinases, phosphatases, and binding proteins called arrestins. Almost all receptors undergo desensitization when the cell is exposed to extracellular signals for prolonged periods (Heldin et al. 2014). Desensitization often involves these proteins. Similarly, GTPases are deactivated by GAPs, and the effects of protein kinases are tightly controlled by protein phosphatases that antagonize them. The activities of effectors such as ACs are controlled by degradation of their products (in the case of $\mathrm{AC}$, the second messenger cAMP by phosphodiesterases). There is a large family of phosphodiesterases that is subject to differential regulation by protein kinases, scaffolds, and by calcium/ calmodulin. Both GAPs and phosphatases can be regulated by protein kinases and scaffolds as well. Thus, every positive signal generator in the cell has a negative counterpart that allows signal dissipation as needed.

\subsection{Signaling Pathways and Networks Can Filter Noise}

The coupling of positive and negative components allows signaling networks to filter noise in their inputs. Thus, low-level or transient signals may activate a few upstream signaling reactions but generate no cellular response (e.g., gene expression). A number of regulatory motifs improve signal-to-noise properties of networks. As described above, positive-feedback loops that function as switches can filter out subthreshold stimuli as noise. Feedforward motifs and interlinked feedback loops can also be configured so that they respond to sustained stimuli but ignore transient stimuli as noise (Brandman et al. 2005). Bi-fans that require activation of both upstream components to produce downstream effects function as AND gates (Fig. 3D). Such a network motif can function as a double coincidence detector that requires multiple upstream signals to activate downstream components. Such a configuration can help prevent activation of downstream events such as transcription when the transcription factor is phosphorylated by only one protein kinase (i.e., only one signal is received) (Lipshtat et al. 2008).

\section{CONCLUDING REMARKS}

Signaling networks in cells produce outputs that are manifested as decisions to perform physiological functions in response to biochemical, electrical, or mechanical stimuli. These outputs are most often controlled by protein kinases in the cytoplasm and near the plasma membrane. These protein kinases phosphorylate and regulate metabolic enzymes, channels, transporters, and components of the cytoskeletal machinery. In the nucleus, the targets are typically transcription factors and proteins that control chromosomal organization and dynamics. The outputs, whether they are production of glucose from glycogen by liver cells in response to epinephrine, firing of neurons in response to neurotransmitters, or hormone-driven changes in gene expression of ovarian cells, reflect both the characteristics (amplitude and duration) of the external signals and information processing within signaling networks. The ability to balance these allows the cell to respond to varying stimuli and return to homeostatic balance.

When signaling components are inappropriately activated or inactivated (e.g., by mutations), however, the information-processing capability of the networks is also altered. This may lead to sustained changes in gene expression that push the cell into a different state, for example, an enhanced rate of proliferation, which is often detrimental and can cause diseases such as cancer (Brugge and Sever 2014). Similarly, bacterial toxins can cause disease by altering components within signaling networks (Orth and Alto 2012). Both examples show how critical it is for us to understand the organization and information-processing capability of signaling networks.

\section{REFERENCES}

* Reference is also in this collection.

Alon U. 2007. Network motifs: Theory and experimental approaches. Nat Rev Genet 8: 450-461.

Bagci EZ, Vodovotz Y, Billiar TR, Ermentrout GB, Bahar I. 2006. Bistability in apoptosis: Roles of bax, bcl-2, and mitochondrial permeability transition pores. Biophys J 90: 1546-1559.

Barabasi AL, Albert R. 1999. Emergence of scaling in random networks. Science 286: 509-512. 
Bar-Sagi D, Hall A. 2000. Ras and Rho GTPases: A family reunion. Cell 103: $227-238$.

Bhalla US, Iyengar R. 1999. Emergent properties of networks of biological signaling pathways. Science 283: 381-387.

Biggs N, Lloyd EK, Wilson RJ. 1986. Graph theory 1736-1936. Clarendon, Oxford.

* Bootman M. 2012. Calcium signaling. Cold Spring Harb Perspect Biol 4: a011171.

Brandman O, Ferrell JE Jr, Li R, Meyer T. 2005. Interlinked fast and slow positive feedback loops drive reliable cell decisions. Science 310: 496498.

* Brugge J, Sever R. 2014. cAMP signaling in cancer. Cold Spring Harb Perspect Biol doi: 10.1101/cshperspect.a006098.

Ferrell JE Jr, Machleder EM. 1998. The biochemical basis of an all-ornone cell fate switch in Xenopus oocytes. Science 280: 895-898.

Gillespie DT. 2007. Stochastic simulation of chemical kinetics. Annu Rev Phys Chem 58: 35-55.

Goldbeter A, Koshland DE Jr. 1981. An amplified sensitivity arising from covalent modification in biological systems. Proc Natl Acad Sci 78: $6840-6844$.

* Green DR, Llambi F. 2014. Cell death signaling. Cold Spring Harb Perspect Biol doi: 10.1101/cshperspect.a006080.

* Heldin C-H, Lu B, Evans R, Gutkind JS. 2014. Signals and receptors. Cold Spring Harb Perspect Biol doi: 10.1101/cshperspect.a005900.

* Hemmings B. 2012. PI3K signaling. Cold Spring Harb Perspect Biol 4: 011189.

Jordan JD, Landau EM, Iyengar R. 2000. Signaling networks: The origins of cellular multitasking. Cell 103: 193-200.

Lipshtat A, Purushothaman SP, Iyengar R, Ma'ayan A. 2008. Functions of bifans in context of multiple regulatory motifs in signaling networks. Biophys J 94: 2566-2579.

Lipshtat A, Jayaraman G, He JC, Iyengar R. 2010. Design of versatile biochemical switches that respond to amplitude, duration, and spatial cues. Proc Natl Acad Sci 107: 1247-1252.
Mangan S, Alon U. 2003. Structure and function of the feed-forward loop network motif. Proc Natl Acad Sci 100: 11980-11985.

* Morrison D. 2012. MAPK kinase pathways. Cold Spring Harb Perspect Biol 4: a011254.

* Newton AS. 2014. Second messengers. Cold Spring Harb Perspect Biol doi: 10.1101/cshperspect.a005926.

Novak B, Tyson JJ. 1993. Numerical analysis of a comprehensive model of M-phase control in Xenopus oocyte extracts and intact embryos. J Cell Sci 106: $1153-1168$.

* Orth K, Alto N. 2012. Subversion of cell signaling by pathogens. Cold Spring Harb Perspect Biol 4: a006114.

Pieroni JP, Jacobowitz O, Chen J, Iyengar R. 1993. Signal recognition and integration by Gs-stimulated adenylyl cyclases. Curr Opin Neurobiol 3: $345-351$

Pomerening JR, Kim SY, Ferrell JE Jr. 2005. Systems-level dissection of the cell-cycle oscillator: Bypassing positive feedback produces damped oscillations. Cell 122: 565-578.

Pool IdS, Milgram S, Newcomb T. 1989. The small world. Ablex, Norwood, NJ.

* Sassone-Corsi P. 2012. The cyclic AMP pathway. Cold Spring Harb Perspect Biol 4: a011148.

Sha W, Moore J, Chen K, Lassaletta AD, Yi CS, Tyson JJ, Sible JC. 2003. Hysteresis drives cell-cycle transitions in Xenopus laevis egg extracts. Proc Natl Acad Sci 100: 975-980.

Tanaka K, Augustine GJ. 2008. A positive feedback signal transduction loop determines timing of cerebellar long-term depression. Neuron 59: $608-620$

Tyson JJ, Novak B. 2010. Functional motifs in biochemical reaction networks. Annu Rev Phys Chem 61: 219-240.

Weng G, Bhalla US, Iyengar R. 1999. Complexity in biological signaling systems. Science 284: 92-96.

Xiong X. 2013. Signaling pathways that control cell proliferation. Cold Spring Harb Perspect Biol 5: a008904. 


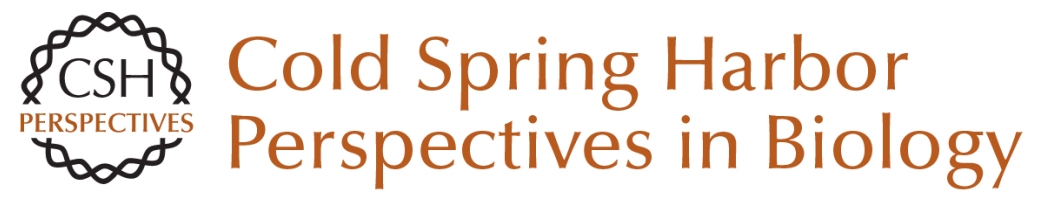

\section{Signaling Networks: Information Flow, Computation, and Decision Making}

Evren U. Azeloglu and Ravi lyengar

Cold Spring Harb Perspect Biol 2015; doi: 10.1101/cshperspect.a005934

Subject Collection Signal Transduction

Cell Signaling and Stress Responses

Gökhan S. Hotamisligil and Roger J. Davis

Protein Regulation in Signal Transduction Michael J. Lee and Michael B. Yaffe

Synaptic Signaling in Learning and Memory Mary B. Kennedy

Vertebrate Reproduction Sally Kornbluth and Rafael Fissore

Signaling in Lymphocyte Activation Doreen Cantrell

Signaling in Muscle Contraction Ivana Y. Kuo and Barbara E. Ehrlich

Toll-Like Receptor Signaling Kian-Huat Lim and Louis M. Staudt

Signaling Pathways that Regulate Cell Division Nicholas Rhind and Paul Russell
Second Messengers

Alexandra C. Newton, Martin D. Bootman and John D. Scott

Signals and Receptors Carl-Henrik Heldin, Benson Lu, Ron Evans, et al.

Cell Death Signaling Douglas R. Green and Fabien Llambi

Signaling Networks that Regulate Cell Migration Peter Devreotes and Alan Rick Horwitz

Signaling Networks: Information Flow, Computation, and Decision Making Evren U. Azeloglu and Ravi lyengar

Signal Transduction: From the Atomic Age to the Post-Genomic Era

Jeremy Thorner, Tony Hunter, Lewis C. Cantley, et al.

Signaling by the TGF $\beta$ Superfamily Jeffrey L. Wrana

Subversion of Cell Signaling by Pathogens Neal M. Alto and Kim Orth

For additional articles in this collection, see http://cshperspectives.cshlp.org/cgi/collection/

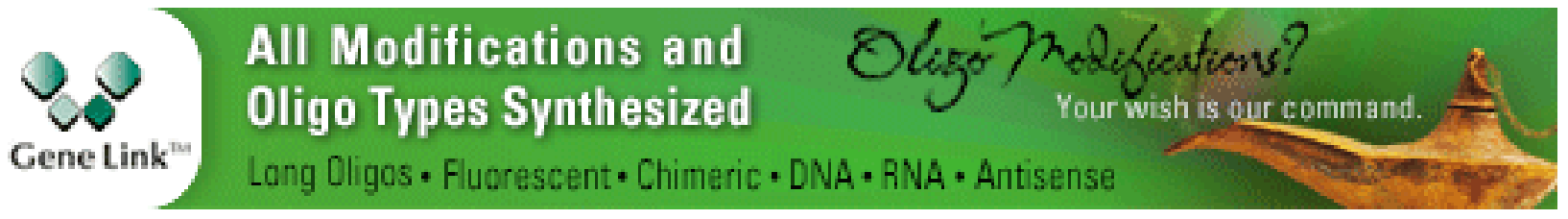

Copyright @ 2015 Cold Spring Harbor Laboratory Press; all rights reserved 